\title{
An improved ADRC and its Application
}

\author{
ZHOU Guo-dong \\ Mechanical and Electrical Engineering Department \\ Hunan Radio and TV University \\ Changsha, China \\ E-mail: zgd79@qq.com.cn
}

\author{
ZHANG Dun-Li \\ Mechanical and Electrical Engineering Department \\ Hunan Radio and TV University \\ Changsha, China \\ E-mail: 11506043@qq.com.cn
}

\begin{abstract}
Electro-hydraulic servo control system has a typical large delay, nonlinear and time-varying characteristics, which is Controlled effectively by using ADRC. To optimize the parameters of ADRC quickly, the paper presents a new ADRC structure of configurable zero point, and analysis the effect of strong interference on the extended state observer, and proposed the selection conditions of ESO parameter. Experiments show. This design can effectively improve the response speed of the system and simplifies the tuning process parameters.
\end{abstract}

Keywords-active disturbances rejection control (ADRC); zero-parameter tunable; electro-hydraulic servo system.

\section{INTRODUCTION}

In the recent years, ADRC is researched widely and deeply by more and more professors, for it has some merits: without system accurate arithmetic model, strong immunity for disturbance, high fitness for control of time-variable system. The structure has been modified from nonlinear to linear. Some intelligent algorithms such as ANN, PSO and

\section{GENERAL CHARGED WITH ADRC MODEL OBJECTS}

\section{A. Generally controlled object model}

Typically, the controlled object model represented by second-order differential equation:

$$
\ddot{y}=-a_{1} \dot{y}-a_{2} y+\omega(t)+b_{0} u=f+b_{0} u \text {. (1) }
$$

Where, $y, y, y$ is the hydraulic cylinder extending the displacement, velocity, acceleration; $u$ is the output controller; $\omega(t)$ is the system load disturbance; $f=f(y, y, \omega(t))$ is the all system disturbance, which including internal and external disturbances system disturbances, is nonlinear to the hydraulic cylinder displacement, velocity, acceleration, load.

\section{B. ADRC's construction}

$\begin{array}{ccc}\text { The } & \text { state } \quad \text { variable } & X\end{array}$ is

SMC are mixed with the ADRC, which make the parameters' tuning more easily. But it will bring the parameters' tuning question of the intelligent control model.

What's more, ADRC is replaced with linear structure, and a large number of application researches show that structure of the linear feedback ADRC for nonlinear uncertain objects still have very good control effect, whose control stability is more easily demonstrated by classical control theory, which compensates for the ADRC lack of control theory to some extent.

The paper proposes a new linear ADRC control structure with an increase of zero adjustable parameters, faster system response speed compared with the traditional ADRC. It changes the way of amount of disturbance compensation control, eliminate the parameter coupling compensation and extends state observer. Electro-hydraulic servo system by following a given experiment showed that adjusting the zero coefficients can effectively speed electro-hydraulic servo system response, and make it easier to adjust the parameters.

The formula (1) is rewritten in the form of the equation of state:

$$
\left\{\begin{array}{c}
\cdot \\
x_{1}=x_{2}, \\
x_{2}=x_{3}+b_{0} u \\
x_{3}=f \\
y=x_{1},
\end{array}\right.
$$

Establish a linear controlled object ADRC, whose extended state observer expression:

$$
\left\{\begin{array}{c}
e=z_{1}-y, \\
\dot{Z}_{1}=z_{2}-\beta_{01} e, \\
Z_{2}=z_{3}-\beta_{02} e+b_{0} u, \\
\cdot Z_{3}=-\beta_{03} e,
\end{array}\right.
$$


Where, $\quad Z=\left[\begin{array}{lll}Z_{1} & Z_{2} & Z_{3}\end{array}\right]^{T}$ is the observation of $\left[\begin{array}{lll}X_{1} & X_{2} & X_{3}\end{array}\right]^{T}$.

Since there is a combination of a variety of ADRC, the article proposes improved linear combinations, which were expressed as:

$$
\left\{\begin{array}{c}
u_{0}=k_{p}\left(r-z_{1}\right)+k_{d}\left(\lambda \dot{r}-z_{2}\right), \\
u=\left(u_{0}-z_{3}\right) / b_{0} .
\end{array}\right.
$$

Where, $\lambda$ is set to a value which is related to the loop zero coefficient of the system. $k_{p}, k_{d}$ are the coefficients of the linear combination, $r, r$ are input to the controlled object and their differential, $u_{0}$ is the amount of control before compensation.

Bringing $\mathrm{u}$ into the control amount of ESO formula (4), we can see that this linear combination eliminate coupling parameters, and make $b_{0}$ only become an enlarged parameter.

When ignored ESO observation error, by the formula (1) (2) can be obtained:

$$
y=\left(f-z_{3}\right)+u_{0} \approx u_{0} .
$$

By the formula (4) can be obtained:

$$
\begin{aligned}
& y=k_{p}\left(r-z_{1}\right)+k_{d}\left(\lambda \dot{r}-z_{2}\right) . \\
& z_{1}=y, z_{2}=y .
\end{aligned}
$$

The formula (7) Laplace transform into equation (6), was charged with the object of closed-loop transfer function expression:

$$
G=\frac{Y}{R}=\frac{\left(k_{p}+\lambda k_{d} S\right)}{S^{2}+k_{d} S+k_{p}} .
$$

Let $k_{d}=2 \xi \omega_{c}, k_{p}=\omega_{c}^{2}, \xi$ is the damping system; $\omega_{c}$ is a natural frequency, when $0<\xi<1$, the system is under damped.

\section{ADRC PERFORMANCE ANALYSIS}

\section{A. Time domain characteristics analysis}

When the input $\mathrm{R}=1 / \mathrm{S}$, that is, the input is a step function, the output expression for $\mathrm{Y}$ :

$$
Y=\frac{\left(\omega_{c}^{2}+2 \lambda \xi \omega_{c} S\right)}{S^{2}+2 \xi \omega_{c} S+\omega_{c}^{2}} \cdot \frac{1}{S}=\frac{1}{S}-\frac{2 \xi(1-\lambda) \omega_{c}+S}{S^{2}+2 \xi \omega_{c} S+\omega_{c}^{2}}
$$

Eq (9) Take Laplace inverse transform to obtain the time domain response of the system:

$$
y(t)=1-e^{-\xi \omega_{c} t} \cos \left(\omega_{d} t\right)-\frac{2 \lambda-\xi}{\sqrt{1-\xi^{2}}} e^{-\xi \omega_{c} t} \sin \left(\omega_{d} t\right) .
$$

Where $\omega_{d}=\omega_{c} \sqrt{1-\xi^{2}}$ referred to the ringing frequency.

The formula (10) shows the step response of the system from the steady state component and a damping component composition $\xi$, the decay rate is mainly determined by $\omega_{c}$, the greater $\omega_{c}$ is, the faster the decay is, the smaller $\xi$ is, the faster the system response. Figure 1 shows the $\xi=0.2,0.4,0.6,0.8$, the coefficient of $\lambda=$ $1.00, \omega_{c}$ is the fixed value, the system step response, $\omega_{d} t$ is the dimensionless time. Obviously, when $\xi$ is small, the system increased time and the peak time is shortened, the greater the overshoot. General admission $0.4<\xi<0.8$, the system response speed and overshoot between compromises.

When $\xi=0.4, \lambda=1.00,0.75,0.55$ and $\xi=0.2, \lambda=1.00$, the system step response curve shown in Figure 2 can be seen, adjust the zero coefficient $\lambda$ becomes small, the rise time and peak time of the system is significantly shortened, the overshoot amount is reduced, which significantly Figure 1 is superior to reduced damping speed up response systems approach.

(8) The description of the system is zero:

$$
S=-k_{p} /\left(\lambda k_{d}\right)=-\omega_{c} /(2 \lambda \xi) \text {. }
$$

Pole of the system:

$$
S=-\xi \omega_{c} \pm j \omega_{c} \sqrt{\xi^{2}-1} \text {. }
$$

When $\xi=0.4, \lambda=1.00,0.75,0.55$ and $\xi=0.2, \lambda=1.00$, the system step response curve shown in Figure 2 can be seen, adjust the zero coefficient $\lambda$ becomes small, the rise time and peak time of the system is significantly shortened, the overshoot amount is reduced, which significantly Figure 1 is superior to reduced damping speed up response systems approach.

(8) The description of the system is zero:

$$
S=-k_{p} /\left(\lambda k_{d}\right)=-\omega_{c} /(2 \lambda \xi) \text {. }
$$

Pole of the system:

$$
S=-\xi \omega_{c} \pm j \omega_{c} \sqrt{\xi^{2}-1} \text {. }
$$

The formula (11) and (12) become small when it can be seen, zero is increased, and the poles of the system does not change, the system response speed is accelerated when $\xi$ changes, system poles and zeros are changed, the adjustment of $\xi$ is more complex. 


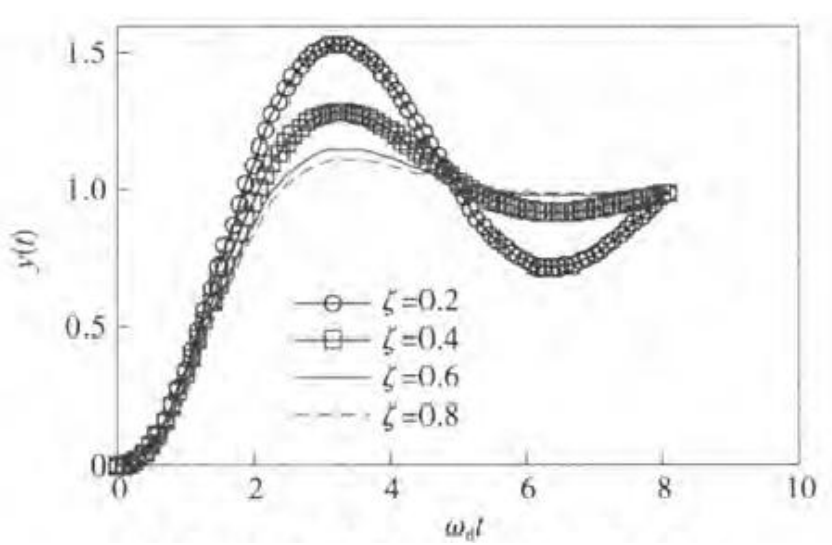

Figure 1. Step response under different damp parameters

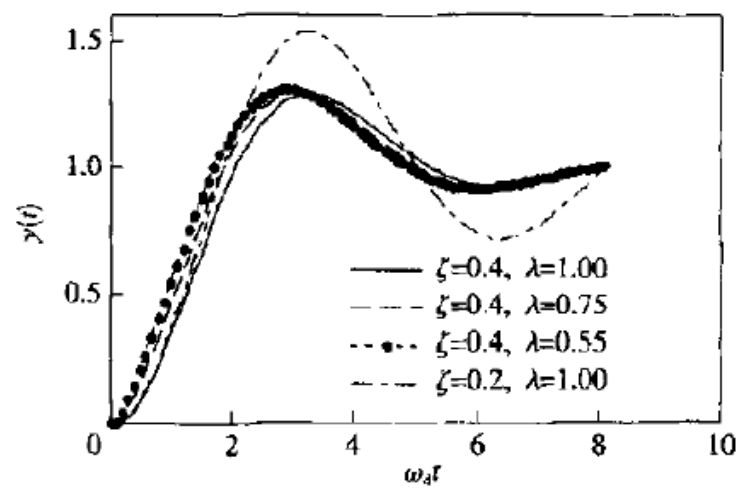

Figure 2. Comparison of system response from the changes of damp parameter $\xi$ and zero-parameter $\lambda$

\section{B. Frequency domain characteristics analysis}

Despite the smaller the $\lambda$ value, the faster system response, even $\lambda$ value is 0 . However, by studying the frequency characteristics of the system, the smaller $\lambda$ found, the system at a high frequency, the greater the phase lags. Figure 3 shows the system's frequency response bode plot when $\mathrm{a}=10,=25,=1.00,=0.10,0.50,1.10$.

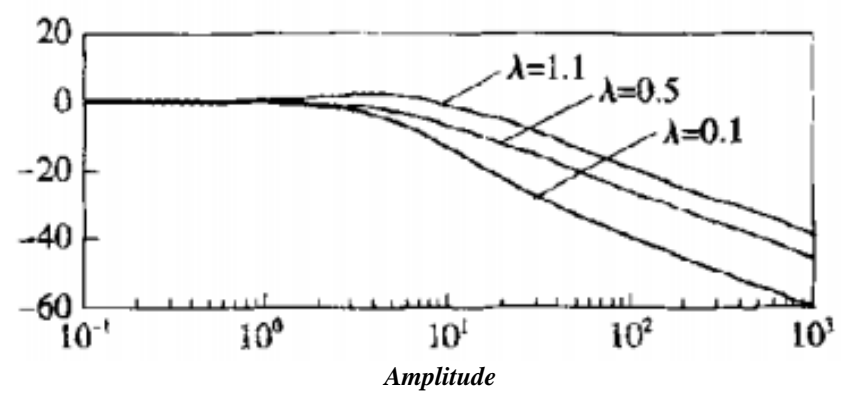



Figure 3. Characteristics in frequency domain under different zero-parameters

As can be seen from Figure 3, when the input frequency $\omega \leq 5 \mathrm{rad} \bullet \mathrm{s}^{-1}, \lambda$ can be reduced as much as possible to increase system response speed, then the phase lag can't be affected.

\section{The impact of noise on ESO}

ESO parameters are designed as follows:

$$
L=\left[\beta_{01} \beta_{02} \beta_{03}\right]=\left[3 \omega_{0} 3 \omega_{0}^{2} \omega_{0}^{3}\right] \text {, }
$$

Where, $\omega_{0}$ is the observed frequency.

ADRC's difficulty lies in the ESO's response speed of its noise amplification is contrary, that the bigger ESO's observing parameters is, the faster response comes, and the amplification of noise is more obvious.

Without considering the given error between the reference value and the output value, considering only the impact of noise on a control amount output, we can get the following express by the formula:

$u_{\delta}=-T_{h} \delta\left(k_{p} \beta_{01}+k_{d} \beta_{02}+\beta_{03} / b_{0}\right)$,

Where, $\mathrm{T}_{\mathrm{h}}$ is the control cycle. As can be seen from the formula (14), $\mathrm{T}$ is smaller, the smaller the effect of noise amplification. So ESO parameters appropriate to reduce the noise of the system is to reduce the interference of effective measures, but doing so will also reduce the response speed of ESO, in the case without noise interference, ESO parameters should take a larger value as possible.

\section{EXPERIMENTAL STUDY}

The paper designs a ADRC block diagram shown in Figure 3, the experiment sinusoidal reference input signal, which can be directly calculated differential, and omits the differential - tracker, where part of a linear combination of adjustable diagonal arrows represent zero .

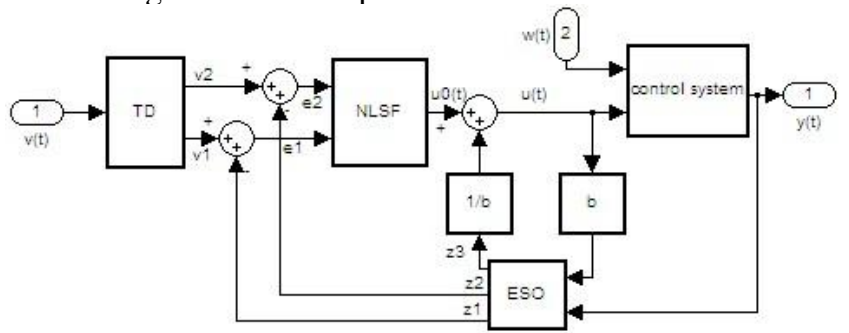

Figure 4. The structure of second order ADRC 
Moog servo valve selection system's D661-G60 series servo valves, displacement sensors built into the servo cylinder, the model for the MTS magnetostrictive displacement sensor company. Platform controller uses the Siemens S7-400, analog input AI module the single-channel conversion time is 52us, resolution 14; AO module single-channel conversion time is 420us; Siemens SCL programming language programming language, the program does not use subroutines computing division and to shorten the control cycle.

\section{CONCLUSION}

The linear control structure is designed in ADRC, which can increase the ADRC's adjustable zero coefficients, and speed up ADRC's response property. By changing the way of linear combination to estimating the disturbance compensation, and eliminating the parameters $\mathrm{b}_{0}$ and ESO coupling, ADRC parameters will be adjusted easily. Experiments show that the parameters can effectively be regulated zero point which can speed up system responsibility, and make it easier for parameters' setting.

\section{REFERENCES}

[1] Han Jingqing. From PID to active disturbance rejection control [J]. IEEE Transactions on Industrial Electronics, 2009,56(3):900-906.

[2] Han Jingqing. Avtive disturbance rejection control tecknique [M]. Beijing: National Defense Industry Press, 2009.

[3] Han yongcheng, Fang Yiming, Li Qiang. Design of sliding model active disturbance rejection controller for hydraulic position servo system[J]. Control Engineering of China, 2007, 14(suppl 1):53-55.

[4] Qiao Guolin, Tong Chaonan, Sun Yikang. Study on mould level and casting speed coordination control based on ADRC with DRNN optimization [J]. ACTA Automatica Sinica, 2007, 33(6):641-648. 